\title{
Interpreting the multi-biomarker disease activity score in the context of tocilizumab treatment for patients with rheumatoid arthritis
}

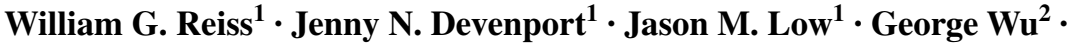 \\ Eric H. Sasso ${ }^{2}$
}

Received: 2 February 2015 / Accepted: 8 May 2015 / Published online: 31 May 2015

(C) Springer-Verlag Berlin Heidelberg 2015

\begin{abstract}
The multi-biomarker disease activity (MBDA) score measures 12 proteins involved in the pathophysiology of rheumatoid arthritis (RA) to assess disease activity (DA). Previous studies demonstrated correlations between MBDA and clinical DA scores with some RA therapies. In this analysis, the relationship between DA and MBDA scores and changes in MBDA component biomarkers were evaluated in tocilizumab (TCZ)-treated patients. Patients from the ACT-RAY study were included in this analysis if they had DA measures and serum collected at pre-specified time points with sufficient serum for MBDA testing at $\geq 1$ visit. Descriptive statistics, associations between outcomes, and percentage agreement between DA categories were calculated. Seventy-eight patients were included and were similar to the ACT-RAY population. Correlations between MBDA score and DAS28-CRP were $\rho=0.50$ at baseline and $\rho=0.26$ at week 24 . Agreement between low/moderate/high categories of MBDA score and DAS28-CRP was observed for $77.1 \%$ of patients at baseline and $23.7 \%$ at week 24. Mean changes from baseline to weeks 4, 12, and 24 were proportionately smaller for MBDA score than DAS28-CRP. Unlike some other MBDA biomarkers, interleukin-6 (IL-6) concentrations increased in most patients during TCZ treatment. Correlations and agreement between MBDA and DAS28-CRP or CDAI scores were lower at week 24 versus baseline. The proportionately smaller magnitude of response observed for MBDA score versus DAS28-CRP may be due to the influence of the increase in
\end{abstract}

William G. Reiss

bill.reiss@ roche.com

1 Genentech, Inc., 1 DNA Way, South San Francisco, CA 94080, USA

2 Crescendo Bioscience, Inc., South San Francisco, CA, USA
IL-6 concentrations on MBDA score. Thus, MBDA scores obtained during TCZ treatment should be interpreted cautiously and in the context of available clinical information.

Keywords Rheumatoid arthritis · Tocilizumab ·

Biomarker · MBDA · Interleukin-6 (IL-6) ·

C-reactive protein (CRP)

\section{Introduction}

Tocilizumab (TCZ) is a recombinant, humanized monoclonal antibody that blocks interleukin 6 (IL-6) from binding to the soluble and membrane-bound IL-6 receptors (IL-6R) and thereby inhibits IL-6-mediated signaling. The clinical and radiographic efficacy of TCZ in patients with rheumatoid arthritis (RA) has been demonstrated in multiple trials [1-5]. TCZ treatment has been shown to have distinctive effects on serum biomarkers $[2,5,6]$. For instance, serum IL-6 concentrations increase during treatment with TCZ [6-8]. In addition, IL-6 is a key stimulator of acute-phase reactants [9], including C-reactive protein (CRP) and serum amyloid A (SAA), and the inhibition of IL-6R by treatment with TCZ leads to a rapid reduction in serum concentrations of CRP and SAA $[1,2,5,10]$.

The use of biologic therapy, alone or in combination with disease-modifying antirheumatic drugs such as methotrexate (MTX), allows for rapid and effective suppression of disease activity (DA) and improvements in clinical signs and symptoms and radiographic outcomes. Treatment guidelines recommend a treat-to-target strategy, with the goal of achieving remission or the lowest attainable disease state $[11,12]$. Clinical assessment tools can aid physicians in reaching this goal by quantifying DA, albeit with varying degrees of subjectivity. Objective measures include serum 
CRP and erythrocyte sedimentation rate (ESR). However, their utility in patients with RA may be limited, as they are often in the normal range for patients with active RA, possibly reflecting physiological differences between individuals $[13,14]$.

A multi-biomarker disease activity (MBDA) blood test has been developed to analyze 12 protein biomarkers, including IL-6, CRP, and SAA, that play a role in the pathophysiology of RA [15]. The test uses an algorithm that combines the biomarker concentrations to produce a DA score on a scale of 1-100. Previous studies of several treatments for RA have demonstrated that MBDA scores correlate with RA disease activity, as measured by the 28-joint Disease Activity Score with CRP (DAS28-CRP) and the Clinical Disease Activity Index (CDAI) [16, 17].

In view of the potential effects that TCZ has on biomarkers included in the MBDA test and DAS28-CRP, the objective of this analysis was to evaluate the association between clinical DA and MBDA scores before and during TCZ treatment and to assess the concomitant effect of TCZ treatment on the component biomarkers of the MBDA test.

\section{Patients and methods}

\section{Participants}

Patients with RA participating in the global ACT-RAY phase 4 study of inadequate responders to MTX [18] were eligible for inclusion in this sub-study if they had provided serum samples at baseline and weeks 4, 12, and 24 of ACTRAY and had DAS28-ESR or DAS28-CRP measured at those visits. Of the patients meeting these criteria, 100 were randomly selected to have MBDA assays performed using frozen serum samples. All patients in the ACT-RAY trial were treated with TCZ $8 \mathrm{mg} / \mathrm{kg}$ every 4 weeks, with $1: 1$ randomization to continue MTX or receive placebo (PBO). This ratio of MTX versus PBO was maintained in the present analysis.

\section{Biomarker assays and clinical assessments}

Measurement of the 12 biomarkers in the MBDA test and calculation of MBDA scores were performed in the development laboratory of Crescendo Bioscience, Inc. (South San Francisco, California) using the Vectra ${ }^{\circledR}$ DA algorithm, as previously described [15, 17, 19]. MBDA scores are reported as integers from 1 to 100 , with the following categories for DA: low $(<30)$, moderate $(30-44)$, and high (>44) [20].

Clinical DA assessments, including swollen joint count (28 joints), tender joint count (28 joints), patient global visual analog scale (VAS), physician global VAS, and high-sensitivity CRP (analyzed centrally), were collected as part of the core study for calculation of DAS28-CRP and CDAI [21, 22]. DAS28-CRP was selected because the MBDA formula is based on this score [17]. CDAI is provided as an alternative DA measure without a laboratory parameter. For the present analyses, conservative DA thresholds were used for DAS28-CRP: low $(\leq 2.67)$, moderate $(2.67$ to $\leq 4.09)$, and high $(>4.09)$ [23]. The DA thresholds used for CDAI were low $(\leq 10)$, moderate (10 to $\leq 22$ ), and high (>22) [24].

\section{Statistical methods}

Descriptive statistics at each visit were calculated for MBDA score, DAS28-CRP, and CDAI. Percentage change from baseline to week 24 was calculated for biomarkers of the MBDA test. Spearman's rank correlation coefficients were estimated, and the corresponding scatter plots were examined to evaluate the association between MBDA scores and clinical measures at each time point. Percentage agreement between DA categories (low, moderate, and high) for each pair of scores (MBDA score and DAS28CRP, MBDA score and CDAI, DAS28-CRP and CDAI) was evaluated at baseline and week 24 .

The analysis of scores at any particular visit was restricted to patients with both an MBDA score and a clinical DA score at that visit. Analysis of change from baseline to a visit was restricted to patients with the required measurements at baseline and that visit. No imputation was performed for data that were missing due to missed visits or incomplete clinical or laboratory information.

\section{Results}

Of the 553 intention-to-treat patients from the ACT-RAY study, 168 patients were eligible for this analysis, 100 were randomly selected, 81 had serum samples available for MBDA testing from at least 1 time point, and 78 contributed scores to the final analysis, although some patients did not have biomarker scores at all time points due to insufficient serum volume. The 78 patients had similar baseline demographics and clinical characteristics compared with those in the larger study population (Table 1) [18]. Mean values (standard deviation) for MBDA score, DAS28-CRP and CDAI for the 48 patients with MBDA scores at baseline were $54.9(17.30), 5.6(0.98)$, and $37.8(12.29, n=47)$, respectively.

Figure 1 presents cross-classifications of patients by MBDA score, DAS28-CRP, and CDAI categories (low, moderate, and high) at baseline and at week 24. Agreements between DAS28-CRP and CDAI were $87.2 \%$ at baseline (prior to initiation of TCZ treatment) and $84.5 \%$ 
Table 1 Demographics and baseline characteristics of the analysis subset and the core study population

\begin{tabular}{lcc}
\hline Characteristic & Analysis subset $(n=78)$ & Core study ITT population [18] $(n=553)$ \\
\hline Female, $n(\%)$ & $64(82.1)$ & $444(80.3)$ \\
Age, mean (SD), years & $50.7(14.40)$ & $53.3(12.67)$ \\
Disease duration, mean (SD), years & $8.5(7.85)$ & $8.2(8.20)$ \\
$<2$ years, $n(\%)$ & $18(23.1)$ & $144(21.2)$ \\
2 to $<5$ years, $n(\%)$ & $17(21.8)$ & $129(26.0)$ \\
5 to $<10$ years, $n(\%)$ & $19(24.4)$ & $163(23.3)$ \\
$\geq 10$ years, $n(\%)$ & $24(30.8)$ & $163(29.5)$ \\
BMI, mean $(\mathrm{SD}), \mathrm{kg} / \mathrm{m}^{2}$ & $25.4(5.00)$ & $26.4(5.16)$ \\
DAS28-CRP, mean $(\mathrm{SD})$ & $5.7(0.91)$ & $5.7(1.0)$ \\
CDAI, mean $(\mathrm{SD})[n]$ & $38.4(12.09)[77]$ & $38.7(12.65)[547]$ \\
\hline
\end{tabular}

$B M I$ body mass index, $C D A I$ Clinical Disease Activity Index, DAS28-CRP 28-joint disease activity score with C-reactive protein, ITT intent to treat, $S D$ standard deviation

\begin{tabular}{|c|c|c|c|c|c|c|c|c|c|}
\hline \multicolumn{5}{|c|}{ Baseline } & \multicolumn{5}{|c|}{ Week 24} \\
\hline & \multirow[b]{2}{*}{$\mathbf{N}$} & \multicolumn{3}{|c|}{ MBDA score } & & \multirow[b]{2}{*}{$\mathbf{N}$} & \multicolumn{3}{|c|}{ MBDA score } \\
\hline & & Low & Moderate & High & & & Low & Moderate & High \\
\hline \multirow{3}{*}{ DAS28-CRP } & Low & 0 & 0 & 0 & \multirow{3}{*}{ DAS28-CRP } & Low & 0 & 12 & 14 \\
\hline & Moderate & 2 & 0 & 1 & & Moderate & 1 & 9 & 17 \\
\hline & High & 3 & 5 & 37 & & High & 0 & 1 & 5 \\
\hline \multicolumn{5}{|c|}{ Disease activity level agreement: $77.1 \%(37 / 48)$} & \multicolumn{5}{|c|}{ Disease activity level agreement: $23.7 \%(14 / 59)$} \\
\hline & & \multicolumn{3}{|c|}{ MBDA score } & & & \multicolumn{3}{|c|}{ MBDA score } \\
\hline & $\mathbf{N}$ & Low & Moderate & High & & $\mathbf{N}$ & Low & Moderate & High \\
\hline \multirow{3}{*}{ CDAI } & Low & 0 & 0 & 0 & \multirow{3}{*}{ CDAl } & Low & 0 & 13 & 19 \\
\hline & Moderate & 0 & 0 & 3 & & Moderate & 1 & 8 & 13 \\
\hline & High & 5 & 5 & 34 & & High & 0 & 1 & 5 \\
\hline \multicolumn{5}{|c|}{ Disease activity level agreement: $72.3 \%(34 / 47)$} & \multicolumn{5}{|c|}{ Disease activity level agreement: $21.7 \%(13 / 60)$} \\
\hline & & \multicolumn{3}{|c|}{ CDAI } & & & \multicolumn{3}{|c|}{ CDAI } \\
\hline & $\mathbf{N}$ & Low & Moderate & High & & $\mathbf{N}$ & Low & Moderate & High \\
\hline \multirow{3}{*}{ DAS28-CRP } & Low & 0 & 0 & 0 & \multirow{3}{*}{ DAS28-CRP } & Low & 24 & 1 & 0 \\
\hline & Moderate & 0 & 0 & 3 & & Moderate & 7 & 20 & 0 \\
\hline & High & 0 & 3 & 41 & & High & 0 & 1 & 5 \\
\hline \multicolumn{5}{|c|}{ Disease activity level agreement: $87.2 \%$ (41/47) } & \multicolumn{5}{|c|}{ Disease activity level agreement: $84.5 \%(49 / 58)$} \\
\hline
\end{tabular}

Fig. 1 Association between MBDA score and clinical disease activity at baseline and week 24. CDAI Clinical Disease Activity Index, DAS28-CRP 28-joint disease activity score with C-reactive protein, $M B D A$ multi-biomarker disease activity. Disease activity thresholds:

at week 24 of TCZ treatment (Fig. 1). Agreement between MBDA score and DAS28-CRP was $77.1 \%$ at baseline and $23.7 \%$ at week 24 . Similar results were observed between MBDA score and CDAI (72.3\% agreement at baseline and $21.7 \%$ agreement at week 24). Further analysis showed that the level of the correlation between MBDA scores and clinical disease assessments also decreased during TCZ treatment. Spearman's rank correlations for DAS28CRP versus MBDA score, CDAI versus MBDA score, and DAS28-CRP versus CDAI were $\rho=0.50,0.19$, and 0.85 , respectively, at baseline and varied between $0.19-0.33$,
MBDA: low (<30), moderate (30-44), high ( $>44)$; DAS28-CRP: low $(\leq 2.67)$, moderate $(>2.67$ to $\leq 4.09)$, high $(>4.09)$; CDAI: low $(\leq 10)$, moderate $(>10$ to $\leq 22)$, high $(>22)$

$0.03-0.24$, and $0.91-0.93$, respectively, for weeks 4 through 24.

Figure 2 presents the means and $95 \%$ confidence intervals (CIs) for change from baseline to weeks 4, 12 and 24 for DAS28-CRP and MBDA scores. Both outcomes decreased over time, demonstrating improvement in DA. However, the MBDA scores decreased proportionately less than the DAS28-CRP. To explore why the MBDA score changed this way during TCZ treatment, the percentage changes in concentration from baseline to week 24 for the individual biomarkers of the MBDA score were examined 


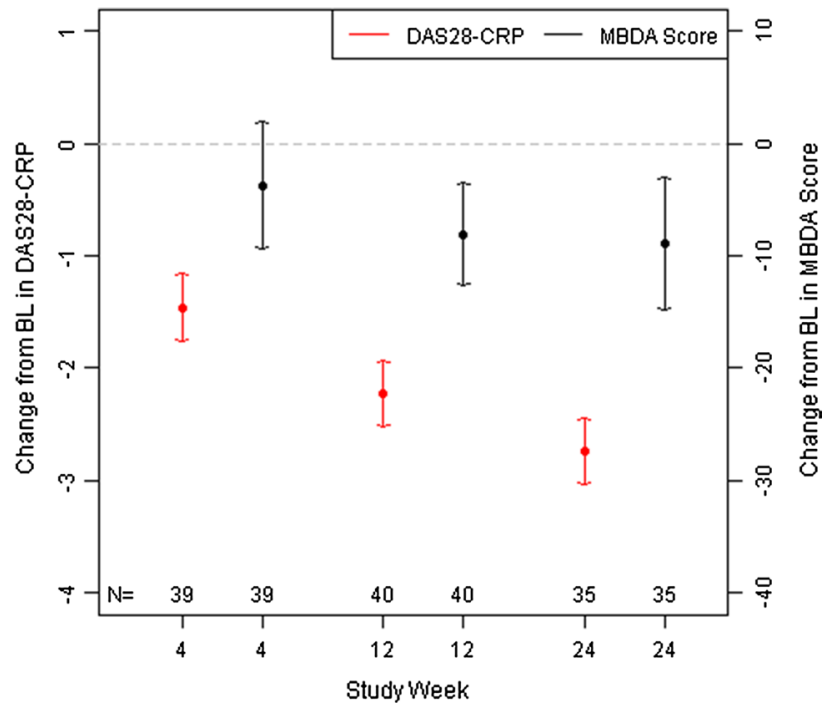

Fig. 2 Mean (95\% CI) change from baseline in DAS28-CRP and MBDA score. MBDA scores (at weeks 12 and 24) and DAS28-CRP (at weeks 4, 12, and 24) were statistically significantly improved from baseline, based on $95 \%$ CI being entirely $<0$. BL baseline, DAS28-CRP 28-joint disease activity score with C-reactive protein, $M B D A$ multi-biomarker disease activity

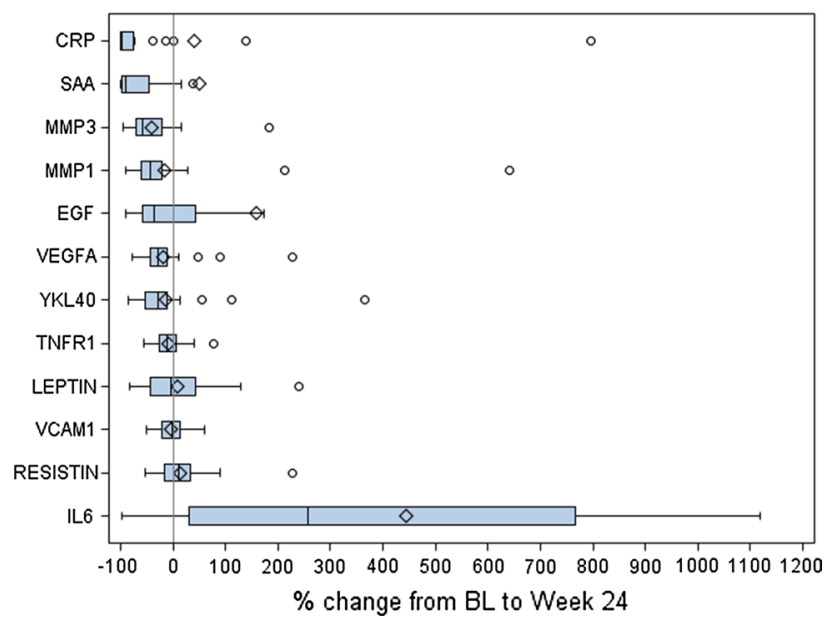

Fig. 3 Percentage changes in MBDA biomarker concentrations from baseline to week 24 . Percentage changes in concentrations of individual biomarkers from baseline to week 24 for patients with MBDA results at both time points $(n=35)$. Biomarkers are sorted by median percentage change from baseline. Each box represents the interquartile range (IQR; 25 th to 75 th percentiles), with the median indicated by a line, the mean indicated by a diamond, and the whiskers extending to the last data values within the $1.5 \times \mathrm{IQR}$ range; circles indicate outliers (defined as points beyond the $1.5 \times \mathrm{IQR}$ ). Seven values with large percentage increases from baseline to week 24 were analyzed, but are not shown: 2 for SAA (1728\% and $2427 \%), 1$ for CRP (3247 \%), 2 for EGF (1840\% and $4000 \%)$, and 2 for IL-6 (1940\% and $2772 \%)$. BL baseline, $C R P$ C-reactive protein, $E G F$ epidermal growth factor, $I L$ interleukin, $M M P$ matrix metalloproteinase, $S A A$ serum amyloid A, TNFR tumor necrosis factor receptor, VCAM vascular cell adhesion molecule, VEGFA vascular endothelial growth factor $\alpha, Y K L-40$ human cartilage glycoprotein 39
(Fig. 3). Median percentage decreases from baseline to week 24 were largest for CRP and SAA. Conversely, IL-6 concentrations showed a large median percentage increase from baseline to week 24 .

\section{Discussion}

In this post hoc analysis, the associations observed between MBDA score and composite clinical DA scores (DAS28CRP and CDAI) were weaker during TCZ treatment than at baseline, prior to TCZ treatment. Similarly, the agreement between categories of MBDA score and clinical DA levels was high at baseline and noticeably reduced after treatment with TCZ for 24 weeks. In addition, MBDA scores did not improve proportionately as much as DAS28-CRP during TCZ treatment, which may have contributed to the low ontreatment correlations between the MBDA and clinical DA scores.

The effect of TCZ on MBDA scores during treatment observed in this analysis may be explained, at least in part, by effects that IL-6 receptor antagonism had on component MBDA biomarkers, specifically increases in IL-6 levels and decreases in CRP and SAA. The algorithm for calculating the MBDA score positively weighs these biomarkers such that higher values result in higher MBDA scores. Our findings suggest that the substantial increases in IL-6 concentrations partially countered the decreases in CRP, SAA, and other biomarkers, thereby contributing to the proportionately smaller improvements observed in the MBDA score, compared with DAS28-CRP and CDAI. This finding is consistent with that of a previous study in Japanese patients with established RA, in which 24 and 52 weeks of TCZ treatment led to increased IL- 6 concentrations in the majority of patients, and to smaller median percentage decreases in MBDA score compared with DAS28-ESR and CDAI [25].

Potential limitations of this sub-study include timing of biomarker sampling, use of concomitant MTX, sample size, and missing data. The timing of serum collection relative to dosing may affect the biomarker concentrations distinctly from the clinical assessments. For example, it is known that CRP levels can fluctuate between TCZ infusions at the $4 \mathrm{mg} / \mathrm{kg}$ dose [1, 3]. However, with the $8 \mathrm{mg} / \mathrm{kg}$ dose used here, decreases in CRP concentration are generally sustained throughout the dosing interval [18]. The present analyses are aggregated results across patients, regardless of randomized treatment; therefore, they do not address the influence of MTX in combination with TCZ versus TCZ alone on the MBDA score or biomarkers. However, previous studies have demonstrated that changes in biomarker serum concentrations (CRP decreases and IL-6 increases) occur during TCZ treatment with or without concomitant 
MTX [5-8, 18, 26]. Finally, this analysis included only a subset of patients from the ACT-RAY trial, and some patients were missing data. Comparison of the demographics and baseline disease characteristics of the present cohort to the full study population demonstrated that the two were quite similar, supporting that the analysis cohort was representative of the full study population. Sensitivity analyses conducted to examine the influence of MBDA scores that were not included in these analyses due to insufficient serum volume for individual biomarker confirmation indicated that conclusions were robust with respect to these values. Additional studies may aid understanding of how these findings generalize to other patients.

In conclusion, this analysis found that MBDA score, DAS28-CRP, and CDAI all detected decreases in DA during 24 weeks of treatment with TCZ. The observed decreases were proportionately smaller for MBDA scores, which underestimated clinical response to TCZ treatment. This finding may reflect the ways that the TCZ mechanism of action generally increases serum concentrations of IL-6 while decreasing CRP and SAA concentrations. Further research is needed to fully understand how to best interpret MBDA scores in patients receiving TCZ. In view of the present findings, MBDA test results should be interpreted cautiously and in the context of all available clinical information to best manage patients with RA during TCZ treatment.

Acknowledgments This study was funded by Genentech, a member of the Roche Group. Genentech sponsored the study and participated in the design of the study as well as in the collection, analysis, and interpretation of the data. Support for third-party writing assistance for this manuscript, furnished by Health Interactions, was provided by F. Hoffmann-La Roche, Ltd.

Conflict of interest W.G. Reiss, J. Devenport and J. Low are employees of Genentech, Inc. E. Sasso and G. Wu are employees of Crescendo Bioscience, a wholly-owned subsidiary of Myriad Genetics, Inc.

\section{References}

1. Smolen JS, Beaulieu A, Rubbert-Roth A et al (2008) Effect of interleukin-6 receptor inhibition with tocilizumab in patients with rheumatoid arthritis (OPTION study): a double-blind, placebo-controlled, randomised trial. Lancet 371:987-997

2. Genovese MC, McKay JD, Nasonov EL et al (2008) Interleukin-6 receptor inhibition with tocilizumab reduces disease activity in rheumatoid arthritis with inadequate response to diseasemodifying antirheumatic drugs: the tocilizumab in combination with traditional disease-modifying antirheumatic drug therapy study. Arthritis Rheum 58:2968-2980

3. Emery P, Keystone E, Tony HP et al (2008) IL-6 receptor inhibition with tocilizumab improves treatment outcomes in patients with rheumatoid arthritis refractory to anti-tumour necrosis factor biologicals: results from a 24-week multicentre randomised placebo-controlled trial. Ann Rheum Dis 67:1516-1523
4. Kremer JM, Blanco R, Brzosko M et al (2011) Tocilizumab inhibits structural joint damage in rheumatoid arthritis patients with inadequate responses to methotrexate: results from the double-blind treatment phase of a randomized placebo-controlled trial of tocilizumab safety and prevention of structural joint damage at one year. Arthritis Rheum 63:609-621

5. Jones G, Sebba A, Gu J et al (2010) Comparison of tocilizumab monotherapy versus methotrexate monotherapy in patients with moderate to severe rheumatoid arthritis: the AMBITION study. Ann Rheum Dis 69:88-96

6. Nishimoto N, Terao K, Mima T et al (2008) Mechanisms and pathologic significances in increase in serum interleukin-6 (IL6) and soluble IL-6 receptor after administration of an anti-IL-6 receptor antibody, tocilizumab, in patients with rheumatoid arthritis and Castleman disease. Blood 112:3959-3964

7. Uchiyama Y, Yoshida H, Koike N et al (2008) Anti-IL-6 receptor antibody increases blood IL-6 level via the blockade of IL-6 clearance, but not via the induction of IL-6 production. Int Immunopharmacol 8:1595-1601

8. Yoshida Y, Sakamoto M, Yokota K et al (2011) Tocilizumab improved both clinical and laboratory manifestations except for interleukin-18 in a case of multiple drug-resistant adult-onset Still's disease. Intern Med 50:1757-1760

9. Gabay C, Kushner I (1999) Acute-phase proteins and other systemic responses to inflammation. N Engl J Med 340:448-454

10. Nishimoto N, Yoshizaki K, Maeda K et al (2003) Toxicity, pharmacokinetics, and dose-finding study of repetitive treatment with the humanized anti-interleukin 6 receptor antibody MRA in rheumatoid arthritis. Phase I/II clinical study. J Rheumatol 30:1426-1435

11. Smolen JS, Landewe R, Breedveld FC et al (2010) EULAR recommendations for the management of rheumatoid arthritis with synthetic and biological disease-modifying antirheumatic drugs. Ann Rheum Dis 69:964-975

12. Singh JA, Furst DE, Bharat A et al (2012) 2012 update of the 2008 American College of Rheumatology recommendations for the use of disease-modifying antirheumatic drugs and biologic agents in the treatment of rheumatoid arthritis. Arthritis Care Res (Hoboken) 64:625-639

13. Pincus T (2005) The American College of Rheumatology (ACR) core data set and derivative "patient only" indices to assess rheumatoid arthritis. Clin Exp Rheumatol 23:S109-S113

14. Clark GH, Fraser CG (1993) Biological variation of acute phase proteins. Ann Clin Biochem 30:373-376

15. Centola M, Cavet G, Shen Y et al (2013) Development of a multi-biomarker disease activity test for rheumatoid arthritis. PLoS One 8:e60635

16. Hirata S, Dirven L, Shen Y et al (2013) A multi-biomarker score measures rheumatoid arthritis disease activity in the BeSt study. Rheumatology (Oxford) 52:1202-1207

17. Bakker MF, Cavet G, Jacobs JW et al (2012) Performance of a multi-biomarker score measuring rheumatoid arthritis disease activity in the CAMERA tight control study. Ann Rheum Dis 71:1692-1697

18. Dougados M, Kissel K, Sheeran T et al (2013) Adding tocilizumab or switching to tocilizumab monotherapy in methotrexate inadequate responders: 24-week symptomatic and structural results of a 2-year randomised controlled strategy trial in rheumatoid arthritis (ACT-RAY). Ann Rheum Dis 72:43-50

19. Eastman PS, Manning WC, Qureshi F et al (2012) Characterization of a multiplex, 12-biomarker test for rheumatoid arthritis. J Pharm Biomed Anal 70:415-424

20. Curtis JR, van der Helm-van Mil AH, Knevel R et al (2012) Validation of a novel multibiomarker test to assess rheumatoid arthritis disease activity. Arthritis Care Res (Hoboken) 64:1794-1803 
21. Prevoo ML, van 't Hof MA, Kuper HH et al (1995) Modified disease activity scores that include twenty-eight-joint counts. Development and validation in a prospective longitudinal study of patients with rheumatoid arthritis. Arthritis Rheum 38:44-48

22. Aletaha D, Nell VP, Stamm T et al (2005) Acute phase reactants add little to composite disease activity indices for rheumatoid arthritis: validation of a clinical activity score. Arthritis Res Ther 7:R796-R806

23. Inoue E, Yamanaka H, Hara $M$ et al (2007) Comparison of Disease Activity Score (DAS)28- erythrocyte sedimentation rate and DAS28- C-reactive protein threshold values. Ann Rheum Dis 66:407-409
24. Aletaha D, Ward MM, Machold KP et al (2005) Remission and active disease in rheumatoid arthritis: defining criteria for disease activity states. Arthritis Rheum 52:2625-2636

25. Hanami K, Hirata S, Tasaka H et al (2013) Behavior of the multibiomarker disease activity (Vectra DA algorithm) score and components in patients with rheumatoid arthritis treated with tocilizumab. Ann Rheum Dis 72(suppl3):391

26. Shimamoto K, Ito T, Ozaki Y et al (2013) Serum interleukin 6 before and after therapy with tocilizumab is a principal biomarker in patients with rheumatoid arthritis. J Rheumatol 40:1074-1081 